Proceedings of XIX International Scientific Conference "New Technologies and Achievements in Metallurgy, Material Engineering, Production Engineering and Physics", Częstochowa, Poland, June 7-8, 2018

\title{
Effect of Stress in Drawn Wires on Magnetization Curves in the Saturation Region
}

\author{
M. SULIGA ${ }^{a}$ AND K. ChWASTEK ${ }^{b, *}$ \\ ${ }^{a}$ Faculty of Materials Processing and Production Technology, Częstochowa University of Technology, \\ al. Armii Krajowej 19, 42-201 Częstochowa, Poland \\ ${ }^{b}$ Faculty of Electrical Engineering, Częstochowa University of Technology, \\ al. Armii Krajowej 17, 42-201 Częstochowa, Poland
}

\begin{abstract}
Magnetic measurements are becoming an important group of nondestructive testing methods for metallurgical products. In the present paper we focus on the possibility to avail of the so-called law of approach to magnetic saturation for determination of variations of mechanical properties (residual stress) in drawn wires. The measured $M=f(H)$ dependences are reasonably well fitted with the simplest Weiss relationship. The value of model parameter $a$ depends significantly on processing conditions.
\end{abstract}

DOI: 10.12693/APhysPolA.135.243

PACS/topics: 75.30.Cr, 75.60.-d, 75.60.Ej

\section{Introduction}

Estimation of residual stresses present in metallurgical products after their final processing is important for safety reasons [1-3]. A number of non-destructive techniques have been developed for this purpose [4-6]. In the present paper we focus on the possibility to estimate the level of residual stresses in drawn wires on the basis of magnetic measurements carried out in the saturation region.

Magnetic properties of ferromagnets in the high field region are described with the phenomenological law of approach to saturation (LAS), which is usually written in the form of a series expansion [7-12]:

$$
M=M_{s}\left(1-\frac{a}{H}-\frac{b}{H^{2}}-\frac{c}{H^{3}}-\cdots\right)+\chi H,
$$

where $M_{s}$ is saturation magnetization, $a, b$, and $\chi$ are constants. The constant $a$ is interpreted as due to inclusions and/or microstress, the constant $b$ - as due to crystal anisotropy [11], whereas $\chi$ is the so-called paramagnetic contribution [12].

There are a number of problems related to this seemingly simple expression. One of the earliest papers on LAS [7] considered just a simplified form of the expression (1), $M=M_{s}\left(1-b / H^{2}\right)$. Cullity and Graham pointed out that the $1 / H$ term would lead to infinite energy at very high field strengths, which implies that either the $a / H$ term is non-physical or valid over a limited field range [11]. The $a / H$ term considered originally by Weiss and Forrer [13] is usually called the magnetic hardness. In Fuller Brown's theory $[8,9]$ it arose from local stress

\footnotetext{
* corresponding author; e-mail: krzysztof .chwastek@gmail.com
}

caused by defects of crystalline lattice, whereas Néel related it to the leakage field from ferromagnetic materials [10]. Grössinger pointed out that there were many experimental proofs confirming a good correlation between the value of $a$ and the level of inhomogeneities present in the sample [14]. Dionne et al. suggested that $a$ might be proportional to the product of saturation magnetization and effective porosity [15]. Up to now it is not clear if magnetic hardness should be accounted for every material.

A wider agreement of the opinions of scientists is met in the case of the second term in the series expansion of relationship (1). It is generally accepted that there exists a proportionality between the values of $b$ and magnetocrystalline anisotropy coefficient $K_{1}$. Holstein and Primakoff pointed out that the coefficient $b$ was conditioned by the plastic deformation of the materials, whereas $c$ was determined by the crystalline properties and elastic state [16]. The order of magnitude and dependence on temperature and elastic condition of the constant $c$, as well as the field variation of the term $c / H^{3}$ indicated that $c$ originated from ferromagnetic anisotropy of crystal grains in the specimen. The latter observation was true no matter whether the anisotropy was a consequence of the natural crystalline properties, or it was intentionally induced. The authors have also found out that $c$ was independent of the metallurgical treatment.

It should be recalled that some authors consider additional fractional power terms of $H$, accounting for the so-called Holstein-Primakoff paraprocess responsible for spin wave amplitude reduction [16] or presence of mean field effects in the material.

In order to determine the values of coefficients appearing in different forms of the expression (1) it is expedient to carry out a transformation of the relationship into an appropriate linearized form (the so-called function rectification) and perform a linear regression fit. 
Such an approach has been recommended by several authors $[14,15,17,18]$. On the basis of physical considerations Utsonomiya et al. derived a quadratic dependence between the value of coefficient $b$ and stress [17]. Subsequently Gimmel and Feller analyzed a linear functional dependence, but for the relationship $a=a(\sigma)$ in the simplified Weiss LAS $M=M_{s}(1-a / H)$ [18]. The fact that values of coefficients appearing in the expression (1) might be correlated with stress opens up a novel possibility to estimate residual stress level in drawn wires from magnetic measurements in the high field region, as pointed out in the report [19].

\section{Measurements and modeling}

The wires examined in the present study have been drawn in industrial conditions using a modern multi-step drawing machine Koch KGT 25/12. The material used in the investigation is $\mathrm{C} 78 \mathrm{DP}$ high carbon $(0.78 \% \mathrm{C}$ ) steel. Before processing the wires have been patented, itched, and phosphored. The drawing process of $5.5 \mathrm{~mm}$ wires to the final diameter $(1.7 \mathrm{~mm})$ was carried out in 12 passes. Magnetic properties of the wires drawn at different speeds and the reference sample i.e. annealed wire have been determined with a vibrating sample magnetometer VSM 7301 from Lakeshore. For modeling purposes we have used the parts of $M=M(H)$ curves for $H \geq 1 \mathrm{kOe}(79.6 \mathrm{kA} / \mathrm{m})$.

The first step was determination of the most suitable and simplest form of the LAS expression for the annealed i.e. stress-free wire. We have checked the following options:

- $M=M_{s}(1-a / H)$,

- $M=M_{s}(1-a / H)+\chi H$,

- $M=M_{s}\left(1-b / H^{2}\right)$,

- $M=M_{s}\left(1-b / H^{2}\right)+\chi H$,

- $M=M_{s}\left(1-a / H-b / H^{2}\right)$.

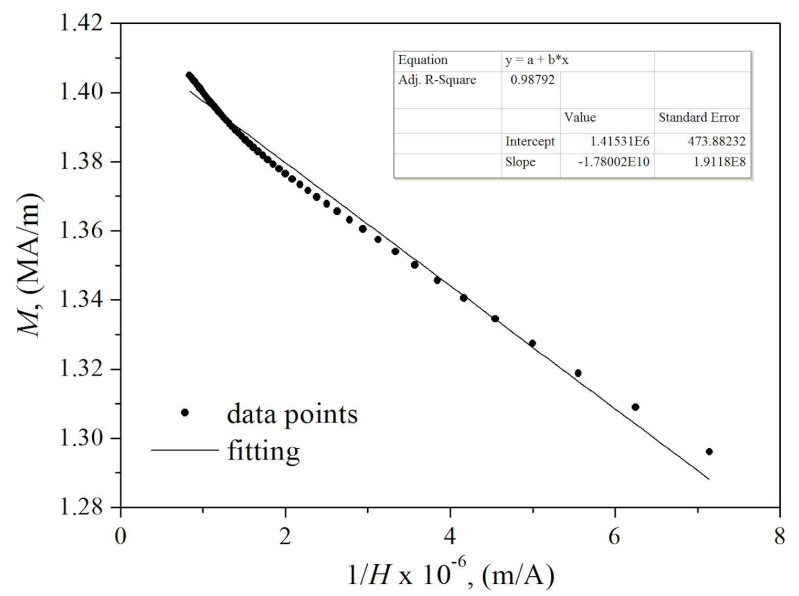

Fig. 1. Fitting of $M$ vs. $1 / H$ using experimental data for the annealed wire.

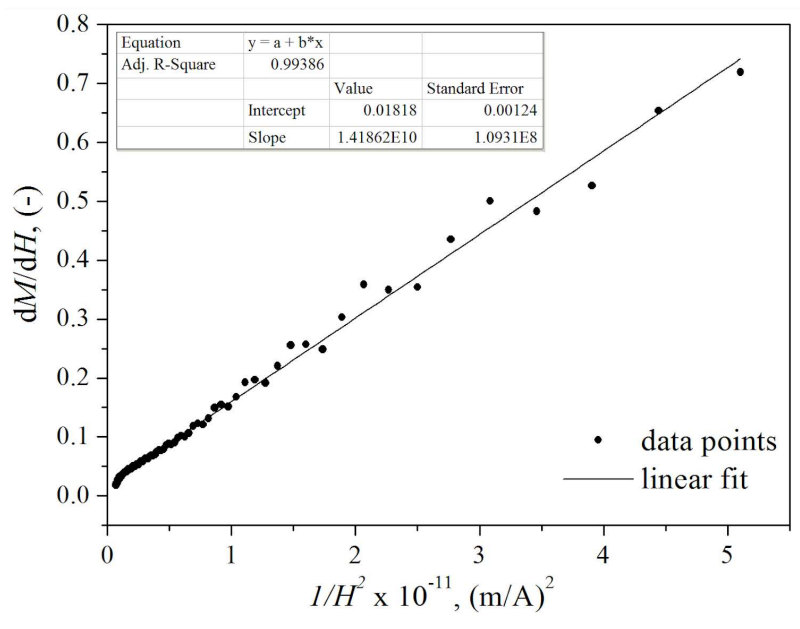

Fig. 2. Fitting of $\mathrm{d} M / \mathrm{d} H$ vs. $1 / H^{2}$ using transformed experimental data for the annealed wire.

The recommended practice to choose the most appropriate relationship for further studies is to carry out a graphical inspection of the rectified relationships [14]. We have found out that for our measurement datasets the term $a / H$ might be dominant in the range of examined field strengths. This can be inferred from Figs. 1 and 2, which represent the relationships $M=f(1 / H)$ and $\mathrm{d} M / \mathrm{d} H=g\left(1 / H^{2}\right)$, respectively. Differential susceptibility presented in the second chart was calculated numerically using fourth-order central differences scheme for the equidistant set of points obtained after smoothing and interpolation of original data. Similar analysis was carried out for other considered relationships, however it has turned out that either the obtained fits were worse or the values of some coefficients were statistically insignificant. In some case we have even obtained non-physical (negative) values. These findings prevented us from repeating Utsonomiya's calculations [17] for our high carbon steel samples, which was the original goal of this contribution.

TABLE I

Values of parameter $a$ and statistical measures for the fits shown in Fig. 3.

\begin{tabular}{c|c|c|c|c|c}
\hline \hline \multicolumn{1}{c|}{ Wire } & Value & Error & N.p. & Reduced $\chi^{2}$ & Adj. $R^{2}$ \\
\hline annealed & 13000 & 200 & 32 & $2.64 \times 10^{7}$ & 0.97226 \\
drawn at $15 \mathrm{~m} / \mathrm{s}$ & 8100 & 100 & 16 & $2.06 \times 10^{6}$ & 0.99734 \\
drawn at $25 \mathrm{~m} / \mathrm{s}$ & 7150 & 100 & 15 & $2.14 \times 10^{6}$ & 0.99656
\end{tabular}

Figure 3 depicts the modelled relationships for the annealed sample and for wires drawn at two speeds i.e. $v=15 \mathrm{~m} / \mathrm{s}$ and $25 \mathrm{~m} / \mathrm{s}$, whereas Table I lists the estimated values of parameter $a$ under different processing conditions and the most relevant statistical measures. Column "Error" denotes the uncertainty in determination of a value, "N.p." is the number of data points, whereas "Reduced $\chi^{2}$ " is the sum of squared deviations between experimental data and theoretical curve. 


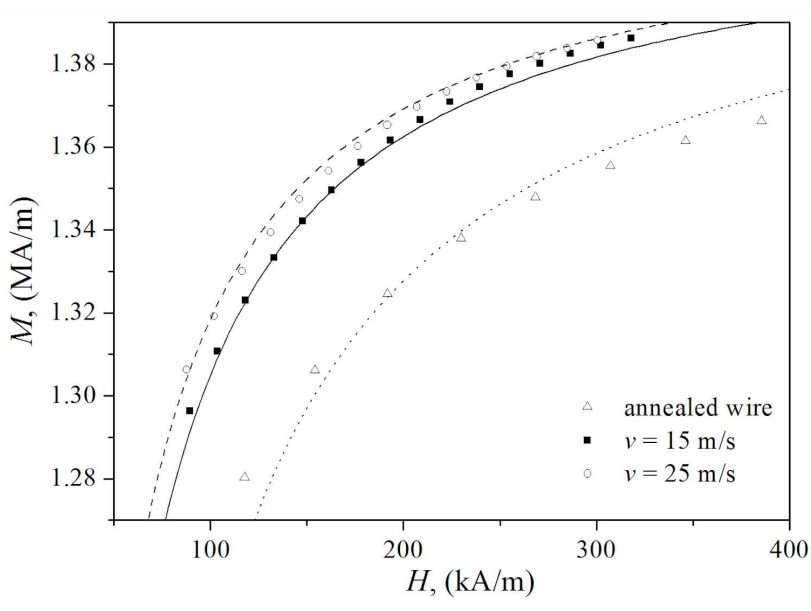

Fig. 3. Fitting of experimental data to the Weiss relationship for different processing conditions.

In the first approximation we have neglected the paramagnetic term $\chi H$ and used the simplest Weiss relationship $M=M_{s}(1-a(\sigma) / H)$ for modeling of magnetization curves of wires after processing. We have found out that the value of saturation magnetization was practically constant (fixed to $M_{s}=1.4 \times 10^{6} \mathrm{~A} / \mathrm{m}$ for the dependences shown in Fig. 3), whereas the value of parameter $a$ was dependent on residual stress inherent in the drawn wires.

\section{Conclusions}

In the present paper we have focused on the possibility to avail of the so-called law of approach to magnetic saturation for determination of mechanical properties (residual stress) of wires subject to different processing conditions. We have pointed out some practical problems related to the LAS relationship. We have carried out measurements of magnetic properties for wires drawn at different speeds and for the annealed wire. We have found out that our measurement data are reasonably well fitted using the simplest LAS form. The model parameter $a$ is found to be dependent on stress. Future work shall focus on determination of an explicit relationship between the level of residual stress and the value of $a$ parameter in a wider range of drawing speeds. Moreover, modeling results shall be verified with other approaches such as the Schepers-Peiter or Sachs-Linicus methods.

\section{Acknowledgments}

The authors are grateful to Ph.D. D.Sc. P. Pawlik, Professor of CUT for help with VSM measurements.

\section{References}

[1] Handbook of Residual Stress and Deformation of Steel, Eds. G. Totten, M. Howes, T. Inoue, ASM International, Materials Park, Ohio 2002.

[2] M. Suliga, Analysis of the Multipass Steel Wire Drawing with High Speed in Conventional and Hydrodynamical Dies, Wyd. WIPMiFS, Częstochowa 2013 (in Polish).

[3] M. Suliga, R. Kruzel, K. Chwastek, A. Jakubas, P. Pawlik, Acta Phys. Pol. A 131, 1114 (2017).

[4] P.J. Withers, Rep. Prog. Phys. 70, 2211 (2007).

[5] G.S. Schajer, C.O. Ruud, in: Practical Residual Stress Measurement Methods, Ed. G.S. Schajer, Wiley, New York 2013.

[6] G.H. Bolognesi Donato, R. Magnabosco, in: Comprehensive Materials Processing, Vol. 2, Ed. S. Hashimi, Elsevier, Amsterdam 2014, p. 219.

[7] N.S. Akulov, Zeit. Phys. 69, 822 (1931) (in German).

[8] W. Fuller Brown, Phys. Rev. 58, 736 (1940).

[9] W. Fuller Brown, Phys. Rev. 60, 139 (1941).

[10] L. Néel, J. Phys. Rad. 9, 184 (1948) (in French).

[11] B.D. Cullity, C.D. Graham, Introduction to Magnetic Materials, IEEE Press, Piscataway 2009.

[12] Hui Zhang, Dechang Zeng, Zhongwu Liu, J. Magn. Magn. Mater. 322, 2375 (2010).

[13] P. Weiss, R. Forrer, Ann. Phys. 10, 279 (1929) (in French).

[14] R. Grössinger, Phys. Status Solidi A 66, 665 (1981).

[15] G.F. Dionne, J.A. Weiss, G.A. Allen, IEEE Trans. Magn. 22, 656 (1986).

[16] T. Holstein, H. Primakoff, Phys. Rev. 58, 1098 (1940).

[17] T. Utsonomiya, H. Nishizawa, K. Kaneta, IEEE Trans. Magn. 27, 3420 (1991).

[18] B. Gimmel, U. Feller, IEEE Trans. Magn. 31, 2241 (1995).

[19] E.W. Collings, Influence of process parameters on the magnetic properties of LTV-processed steel, report submitted to Fermi National Accelerator Laboratory, Batavia, May 1995. 